\title{
ARTIGO
}

Sociologias, Porto Alegre, ano 6, no 11, jan/jun 2004, p. 220-237

\section{Desafios das Ciências Sociais no desenvolvimento científico e tecnológico contemporâneo ${ }^{1}$}

nicialmente gostaria de expressar meu imenso prazer e, ao mesmo tempo, destacar a grande responsabilidade de falar sobre "Os Desafios das Ciências Sociais no Desenvolvimento Científico e Tecnológico Contemporâneo", nesta sessão comemorativa dos 30 anos da Pós-Graduação em Sociologia e Ciência Política da UFRGS. Trata-se de programas que vêm mantendo, ao longo dos anos, uma série de relações institucionais com o Programa de Pós-Graduação em Sociologia da UnB, do qual faço parte, através de pesquisas e publicações conjuntas sobre várias temáticas tais como: educação, ciência, violência, trabalho, entre outras.

Entretanto, gostaria de enfatizar que se trata de comemorar uma construção institucional, isto é, uma obra coletiva e consolidada, o que é mais ainda valioso. Se a Sociologia, segundo o nosso clássico Durkheim, é a ciência que busca conhecer a gênese e o funcionamento das instituições, como não festejar uma instituição que tem analisado com regularidade e

* Professora do Programa de Pós-Graduação em Sociologia da Universidade de Brasília. Pós-dourorado na Ecole des Hautes Etudes en Sciences Sociales (EHESS) em Paris.

1 Conferência proferida nas atividades de comemoração dos 30 anos do Programa de Pós-Graduação em Sociologia da UFRGS. 
qualidade as instituições políticas, educacionais, científicas e as suas transformações, sobretudo num país no qual as descontinuidades das políticas nem sempre favorecem as instituições?

Em sendo uma construção institucional, deve-se a muitas pessoas, professores, alunos e funcionários que se dedicaram a essa obra: uns mais temporariamente e outros continuamente, ou mesmo, durante toda a sua vida acadêmica. Tal construção possibilitou o surgimento de lideranças intelectuais e de grupos de pesquisa formados por professores e estudantes de graduação e pós-graduação.

Também merece destaque a diversidade de formação dos seus professores (universidades norte-americanas, francesas, alemãs, brasileiras, entre outras) e de linhas de pesquisa. Contribuíram para essa construção institucional a política de pós-graduação da CAPES, caracterizada por sua estabilidade e avaliação, e a política de fomento do CNPq, por meio de concessão de bolsas e auxílios, embora nem sempre de uma forma regular e suficiente.

Dessa maneira, a preocupação com a qualidade, a pluralidade teórico-metodológica e temática, com a solidez do conhecimento e, finalmente, a capacidade de acompanhar os temas emergentes fizeram a consolidação desse programa de pós-graduação. É isso que devemos comemorar.

No entanto, cabe-me analisar sociologicamente o contexto amplo do desenvolvimento científico e tecnológico que torna possível e, ao mesmo tempo, limita a produção do conhecimento em Ciências Sociais. Assim, pretendo apresentar aqui sucintamente algumas idéias de estudos contemporâneos sobre as novas tendências do desenvolvimento científico e tecnológico e abordar as políticas brasileiras recentes em ciência e tecnologia sob a ótica dessas idéias. Em seguida, serão mostrados alguns resultados de pesquisa que podem indicar essas novas tendências e, finalmente, vou discutir os desafios das Ciências Sociais diante desse quadro. 


\section{Novas tendências do desenvolvimento científico e tecnológico}

Merton, o grande teórico da Sociologia da Ciência, ante as interferências de um Estado autoritário nazista na produção do conhecimento, propunha uma autonomia da ciência, no sentido de os cientistas ignorarem, em suas pesquisas, todas as considerações que não fossem ligadas ao progresso do conhecimento, além de referir-se ao caráter universal e público da ciência.

Bourdieu, embora considerasse o campo científico um lugar de disputa pelo monopólio da autoridade científica definida como capacidade técnica e como poder social, no sentido de falar e agir legitimamente, não deixava de considerar sua relação com outros campos sociais. No entanto, afirmava esse autor, o campo científico tem suas próprias regras e lógica de funcionamento. As questões relativas a outros campos com os quais interage são refuncionalizadas e traduzidas pela lógica do próprio campo. Nessa perspectiva, há autonomia com interação.

E hoje, como essas questões vêm sendo abordadas? Através da análise da emergência de um novo modo de produção do conhecimento por Gibbons, do modelo da tripla hélice de Etzkowitz e Leydesdorff, da idéia de redes sociotécnicas, elaborada por Latour e Woolgar e do conceito de arenas transepistêmicas segundo Knorr Cetina.

Gibbons e seus colaboradores, em obra de 1994, referem-se ao surgimento de um novo modo de produção do conhecimento. Esse novo modo de produção do conhecimento situa-se num contexto de aplicabilidade, ou seja, são desenvolvidas pesquisas a partir da necessidade de resolver problemas práticos e não apenas de interesses cognitivos, como na pesquisa básica. Também esse novo modo de produção do conhecimento é mais transdisciplinar do que disciplinar, pois, se o conhecimento é produzido num contexto de aplicação e não apenas com a intenção de acumulação do conhecimento na área, muitas vezes, o problema a ser solucionado através do conhecimento exige 
que disciplinas complementares trabalhem a seu respeito.

Além disso, o novo modo de produção do conhecimento pressupõe uma heterogeneidade institucional, pois se desenvolve não apenas na universidade, mas em várias organizações, entre as quais empresas multinacionais, empresas de redes, empresas pequenas de alta tecnologia, laboratórios de pesquisa, ONGs, bem como em programas de cooperação nacional e internacional de pesquisa. Há um aumento do número de locais onde o conhecimento pode ser produzido (não apenas nas universidades). As relações entre os locais se dá das mais variadas formas (eletrônica, organizacional e informalmente) através do estabelecimento de redes de comunicação.

Esse novo modo de produção de conhecimento não é orientado apenas para os pares, mas também para os não produtores de conhecimento, implicando uma maior responsabilidade social. Nos anos mais recentes, houve aumento da consciência pública sobre meio ambiente, saúde, reprodução, entre outros, o que estimula a produção do conhecimento já dentro desses novos moldes.

O novo modo de produção do conhecimento provoca mudanças na pesquisa desenvolvida tradicionalmente nas universidades, que era mais disciplinar e se realizava, sobretudo, num contexto acadêmico orientado pelo próprio processo de conhecimento e não por sua utilidade econômica ou social. No entanto, a consolidação do novo modo de produção do conhecimento não implica necessariamente a substituição do antigo.

Essas novas tendências se vinculam, segundo Gibbons e seus colaboradores, à massificação da educação e da pesquisa (ensino superior e pósgraduação), pois com o crescimento do número de pessoas formadas em pesquisa, estas não ficam apenas dentro da estrutura universitária e disciplinar. Algumas vão trabalhar em laboratórios governamentais ou de empresas. Conseqüentemente, o número de locais com pessoas competentes em pesquisa aumenta. Também se deve ao processo de globalização, que 
aumenta a competitividade internacional e faz com que as empresas precisem produzir inovações tecnológicas, necessitando do conhecimento especializado e de arranjos cooperativos com as universidades, o governo e outras empresas. Essas inovações também se relacionam ao processo de democratização da sociedade, à medida que, cada vez mais, a imprensa, as ONGs e os movimentos sociais procuram influenciar para que a produção científica tenha uma maior responsabilidade social. A própria revolução científica e tecnológica e, sobretudo, o desenvolvimento dos meios de comunicação e informação possibilitam o processo de democratização e a interação de diferentes atores e de diferentes instituições no processo de produção do conhecimento.

Já Etzkowitz e Leydesdorf ressaltam que um novo contrato social entre a universidade e a sociedade está sendo negociado, diferentemente do antigo. Antes, havia um modelo linear de inovação, que presumia contribuições da academia para o setor produtivo somente a longo prazo. Hoje, o modelo da tripla hélice visa analisar a dinâmica das relações entre universidade, empresas e governo, abordando, sobretudo, as interfaces que podem ser desenvolvidas e as relações de comunicação entre elas.

Novos códigos e padrões estão sendo desenvolvidos em todas as interfaces. Por exemplo, a ciência passa a ser valorizada não apenas enquanto procura da verdade, mas também a partir de uma perspectiva de utilização. Políticas governamentais são desenvolvidas como suporte de processos de inovações, sistemas legais são estabelecidos e as indústrias procuram adaptar-se às novas opções tecnológicas. Muitas universidades, públicas e privadas, criam mecanismos para possibilitar as relações entre academia e indústria: escritórios para patenteamento e licenciamento tecnológicos, centros de pesquisa interdisciplinares com participação industrial, parques tecnológicos e incubadoras de empresas.

Ou seja, as instituições estão incluídas em transições que são causadas pelas interações entre as hélices. Nos países em desenvolvimento, 
segundo esses autores, as ONGs também têm importância. Elas se têm mostrado capazes de influenciar a introdução de tecnologias na sociedade, através de organização própria e de alianças com os movimentos sociais. A tese da "tripla hélice" implica que o paradigma de pesquisa nos estudos de inovação incorpora "um modo de rede", incluindo a relação com uma pluralidade de ambientes.

A própria revolução científica e tecnológica e especialmente o desenvolvimento dos meios de comunicação e informação, bem como a democratização da sociedade, possibilitam a interação entre diferentes atores e instituições no processo de produção do conhecimento através de redes.

Já Latour e Woolgar, falam das redes sociotécnicas que se constituem num conjunto complexo e múltiplo de pesquisadores, cientistas, técnicos das agências financiadoras, empresários, membros da sociedade que, direta ou indiretamente, influenciam na elaboração dos problemas. A tecnociência tem características de redes, com laçadas e nós, fios e malhas, significando que a rede envolve não só aqueles que nela estão inseridos, mas também milhares de pessoas que não fazem ciência, mas dependem dela no seu dia a dia, ou nela depositam seus créditos, ou ainda a têm como ponto de referência para o julgamento de seus pensamentos e ações.

Essa idéia também é explicitada por Knorr Cetina através da noção de "arena transepistêmica", em contraposição à noção de comunidade científica, que se limita a estudar apenas os mecanismos de integração circunscritos à especialidade científica e a um determinado paradigma. Quando se passa a estudar o desenvolvimento da ciência a partir do local da pesquisa (do laboratório), é a noção de "arena transepistêmica" que se torna pertinente, afirmando que são as arenas de ação dentro das quais a pesquisa científica e tecnológica se realiza, incluindo cientistas e não-cientistas, reunindo argumentos e conceitos de natureza técnica e não-técnica. Significa dizer que as interações entre os membros de um grupo de especialistas com outros cientistas e com agências de fomento, empresas, governo e ONGs não se resu- 
mem a conteúdos apenas cognitivos, mas também de ordem social.

Dessa forma, pode-se verificar que a inserção cada vez maior da ciência e da tecnologia na sociedade é uma questão bastante discutida na atualidade, evidenciando a complexidade da relação entre autonomia da ciência e a idéia de ciência e da tecnologia enquanto estratégias socioeconômicas. Vou mostrar como esta questão se tem colocado na política recente de ciência e tecnologia (1998/2002).

\section{Política científica e tecnológica recente}

Quando são considerados alguns planos e programas dos últimos anos, em diferentes fases de implantação e implementação, a análise de seus documentos e de editais indica um estímulo maior à pesquisa aplicada e tecnológica, à constituição de projetos cooperativos entre as universidades e outras instituições públicas e privadas e à multidisciplinaridade, semelhantemente aos modelos antes descritos, elaborados sobretudo nos países desenvolvidos.

O Plano Plurianual do MCT (PPA) 2000-2003, os Fundos Setoriais e o novo programa Institutos do Milênio apresentam características que estimulam a emergência dessas tendências. Entre os objetivos do PPA 2000-2003 estão:

a) consolidar, expandir e aprimorar a base nacional de ciência e tecnologia;

b) viabilizar um efetivo sistema nacional de inovação, daí a necessidade de alocação de uma lei de inovação (em preparação), a fim de estimular as empresas privadas a investirem em inovação tecnológica;

c) preparar o país para os desafios da sociedade da informação e do conhecimento;

d) promover a capacitação científica e tecnológica em setores estratégicos para o desenvolvimento do país; 
e) inserir a ciência e tecnologia nas estratégias de desenvolvimento social. Apresenta quinze programas temáticos, tais como Segurança Nuclear, Aplicações Nucleares na Área Médica, Biotecnologia e Recursos Genéticos, Ciência e Tecnologia para o Agronegócio, Atividades Espaciais, Ciência e Tecnologia para a Gestão de Ecossistemas, Desenvolvimento Tecnológico na Área Nuclear, Climatologia, Meteorologia e Hidrologia, Fomento ao Desenvolvimento Tecnológico no Setor Petrolífero, Produção de Componentes e Insumos para a Indústria Nuclear de Alta Tecnologia, Fomento à Pesquisa em Saúde, Mudanças Climáticas e Produção de Equipamentos para a Indústria Pesada, Segurança Nuclear, Sociedade da Informação, todos com um componente aplicado e tecnológico acentuado. Esses programas temáticos estão em diferentes fases de implementação, tendo ações em vários órgãos do governo. A maioria das ações já existiam e foram deslocadas para o PPA.

Já os Fundos Setoriais são, de fato, uma nova forma de financiamento para a ciência e, sobretudo, para a tecnologia. A intenção é de ter recursos estáveis e desvinculados do orçamento geral da União. Os recursos desses Fundos vêm de receitas fiscais extra-orçamentárias, do faturamento das empresas (na sua maioria já privatizadas) atuantes em importantes setores da economia, que serão utilizados para financiar projetos de pesquisa naqueles setores: petróleo, energia, transportes, telecomunicações, mineração, espaço, recursos hídricos, minerais, tecnologia da informação, biotecnologia, agronegócio, aeronáutica e saúde. Mas há dois fundos mais gerais: $20 \%$ do total desses recursos vão para a infra-estrutura e podem apoiar todas as áreas de conhecimento, e ainda, o Fundo Verde-amarelo, que é destinado à inovação tecnológica em programas cooperativos entre universidades, centros de pesquisa e empresas. Além disso, parte dos recursos (30\%) será destinada às regiões menos desenvolvidas, essencial para desconcentrar a pesquisa no país.

Esses fundos podem representar mudanças em relação aos mecanis- 
mos tradicionais de financiamento, pois pressupõem uma maior estabilidade de financiamento, interação entre comunidade e setor produtivo, gestão orientada por resultados, articulação do MCT com outras áreas do governo e aumento da indução da pesquisa em ciência e tecnologia, embora possam ocorrer muitos problemas na sua gestão, em função de conflitos entre atores e instituições diversificadas.

Finalmente, os Institutos do Milênio foram implantados, na forma de redes de pesquisa, segundo prioridades estabelecidas pelo MCT. A inovação desse programa se refere ao processo, pois pretende consolidar a articulação de redes de pesquisa, e ao produto, pois o conhecimento deve contribuir para o aumento da competitividade da economia brasileira e para a resolução de grandes problemas nacionais de cunho social.

O objetivo principal do programa é aumentar a competência científica brasileira em áreas importantes da ciência e da tecnologia, mediante o apoio e a capacitação de centros de pesquisa e laboratórios de alta qualidade científica e/ou atuantes em áreas estratégicas para o desenvolvimento do país. Estes institutos estão divididos em dois grupos. Dois terços dos recursos financiam um primeiro grupo, que é aberto a todas as áreas de conhecimento, mas cujos objetivos devem estar voltados para a aplicabilidade. Já um terço dos recursos destina-se a um segundo grupo, que deve atuar em áreas estratégicas. A operacionalização do programa prevê a articulação de redes científicas e tecnológicas e a integração com centros internacionais de pesquisa, o estímulo à inovação tecnológica, a promoção de projetos inter e multidisciplinares e a desconcentração do conhecimento em benefício das regiões brasileiras com menor desenvolvimento científico e tecnológico.

Foram selecionados os seguintes institutos do Grupo 1: Avanço Global e Integrado da Matemática Brasileira; Fábrica do Milênio; Núcleo de Estudos Costeiros; Instituto do Milênio para Evolução de Estrelas e Galáxias na Era dos Grandes Telescópios; Integração de Melhoramento Genético, 
Genoma Funcional e Comparativo de Citrus; Estratégias Integradas para Estudo e Controle da Tuberculose no Brasil; Mudanças Causadas pelo Uso do Solo na Amazônia; Instituto de Informação Quântica; Instituto do Milênio de Materiais Complexos; Instituto de Nanociências; Água: uma visão mineral; Instituto Multidisciplinar de Materiais Poliméricos; Instituto de Investigação em Imunologia; Bioengenharia Tecidual: Terapias Celulares para Doenças Crônico-Degenerativas; Rede de pesquisa em Sistema em Chip, Microssistema e Nanoeletrônica.

Do Grupo 2, foram constituídos: Instituto do Milênio do Semi-Árido: Biodiversidade, Bioprospecção e Conservação de Recursos Naturais e Instituto do Milênio de Oceanografia: uso e apropriação de recursos costeiros.

De uma forma geral, a política científica e tecnológica recente sinaliza muitas das novas tendências da produção do conhecimento (a aplicabilidade, as redes, a interação com o setor produtivo, a multidisciplinaridade), incorporando ações que já eram desenvolvidas pelo CNPq, em programas especiais que atendem áreas estratégicas, como o PADCT, o RHAE, entre outros. No entanto, deixa uma vertente mais aberta, através dos editais universais de auxílio à pesquisa que financiam projetos para todas as áreas. Além disso, o Programa de Apoio a Núcleos de Excelência (PRONEX) tem como objetivo a consolidação do processo de desenvolvimento científico e tecnológico através de apoio a núcleos de pesquisadores com comprovada competência técnico-científica e tradição na sua área de atuação, tal como é a tradição dos programas básicos que se destinam ao atendimento das demandas nas diversas áreas do conhecimento, mediante critérios de mérito e/ou excelência.

Embora tenha analisado sobretudo os programas do CNPq/MCT, a política de pós-graduação da CAPES também revela essas novas tendências através do estímulo à diversificação do modelo de pós-graduação, visando a formação de acadêmicos, mas também de outros profissionais qualificados, como é o caso da criação dos mestrados profissionalizantes e do incentivo 
aos cursos de pós-graduação lato sensu (Sobral, 1997).

Pode-se concluir que, de fato, essa política científica e tecnológica revela a implementação de um "modelo misto de desenvolvimento científico e tecnológico" (Sobral \& Trigueiro, 1994), que procura associar dois tipos de procedimento: o desenvolvimento impulsionado pelas demandas da própria ciência, ou seja, pelo mercado científico e aquele orientado pelas demandas econômicas e sociais ou pelo mercado econômico e social, talvez no sentido de uma combinação entre o modo mais acadêmico e o chamado novo modo de produção do conhecimento. Dessa forma, preserva-se a autonomia da ciência, mas são enfatizadas necessidades da sociedade, sobretudo de ordem econômica, o que não é totalmente uma novidade, tendo em vista que uma carta de Pasteur ao Ministro da Instrução Pública, em agosto de 1864, citada por Latour, já revela essa tendência:

O vinho constitui uma das maiores riquezas agrícolas da França. O valor deste produto de nosso solo foi acrescido pelo tratado de comércio com a Inglaterra. Há cinco anos eu me dedico aos estudos sobre fermentação. A fermentação alcoólica sob a qual se baseia a fermentação de vinhos tem me ocupado particularmente... O objetivo desta carta é submeter a Vossa Excelência a utilidade de meu projeto, assim como a demanda de recursos que cobririam a sua execução... Mas eu sou o primeiro a não ter ilusão sobre as conseqüências imediatas de meus estudos. A aplicação dos resultados da ciência para a indústria é sempre lenta. Minhas pretensões atuais são modestas. Eu quero chegar a conhecer melhor a planta criptogâmica que é a única causa da fermentação do vinho (Latour, 1995, p. 17-18).

Ou seja, Pasteur cria uma demanda econômica e social possível para a sua pesquisa, tendo em vista a necessidade de financiamento, mas se compromete, de fato, com a produção de conhecimento que poderá, no 
futuro, tornar-se útil para a sociedade.

Pode-se então constatar que o equilíbrio entre a necessária autonomia da ciência e os objetivos econômicos e sociais de um país é uma questão sempre pertinente. Sendo assim, a política em ciência e tecnologia pode considerar a demanda espontânea da comunidade científica e tecnológica brasileira, preservando sua autonomia e mesmo seus vínculos com o cenário científico internacional, mas também o estabelecimento de prioridades temáticas definidas, levando-se em conta demandas e interesses ligados à produção do conhecimento de diferentes segmentos sociais.

\section{Resultados da pesquisa}

Em recente pesquisa ${ }^{2}$ que ainda está em desenvolvimento, cujo objetivo é identificar possíveis convergências entre resultados de ações de fomento do CNPq e as prioridades temáticas estipuladas pelo PPA do MCT (2000-2003), há também indicações desse modelo misto de desenvolvimento científico e tecnológico.

Para fins dessa pesquisa, são considerados como resultados de ações de fomento do CNPq: a) os relatórios de pesquisa de bolsistas do Programa Institucional de Bolsas de Iniciação Científica - PIBIC, elaborados em 2000; b) as dissertações de mestres ex-bolsistas do CNPq, defendidas no mesmo ano de 2000; c) as teses de doutores ex-bolsistas do CNPq, defendidas em 2000; d) os projetos de pesquisa de bolsistas de produtividade que receberam bolsa em 2000.

São consideradas como prioridades temáticas as questões, ou temas, objetivos, ou metas, ou ações expressas nos documentos relativos a treze

2 “O Fomento à Pesquisa do CNPq e os Programas do PPA-MCT" é um projeto que está sendo desenvolvido pelo NESUB (Núcleo de Estudos sobre Ensino Superior) da UnB, sob a minha coordenação e dos professores Carlos Benedito Martins e Jacques Velloso envolvendo consultores e bolsistas de diferentes áreas de conhecimento.

3 O PPA tem quinze programas temáticos mas apenas treze foram analisados pois os programas C e T para o Setor Aeronáutico e Produção de Componente e Insumos para a Indústria Nuclear e de Alta Tecnologia não foram implementados. 
programas temáticos prioritários do MCT já citados anteriormente. ${ }^{3}$

A partir de resultados preliminares desse estudo realizado em seis grandes áreas do conhecimento, ${ }^{4}$ (Ciências Agrárias, Biológicas, Exatas e da Terra, Humanas, Saúde e Sociais Aplicadas), em relação a treze programas prioritários do PPA (Aplicações Nucleares na Área Médica, Biotecnologia e Recursos Genéticos, Ciência e Tecnologia para o Agronegócio, Ciência e Tecnologia para Ecossistemas, Climatologia, Meteorologia e Hidrologia, Desenvolvimento Tecnológico para a Área Nuclear, Fomento ao Desenvolvimento Tecnológico no Setor Petrolífero, Fomento à Pesquisa em Saúde, Mudanças Climáticas, Atividades Espaciais, Produção de Equipamentos para Indústria Pesada, Segurança Nuclear e Sociedade da Informação), percebese um alto grau de convergência dos temas de algumas grandes áreas de conhecimento, sobretudo com os programas que lhes são mais próximos: Ciências Agrárias com o Programa de Ciência e Tecnologia para o Agronegócio, Ciências Biológicas com o Programa de Biotecnologia e Recursos Genéticos, Ciências da Saúde com o programa de Fomento à Pesquisa em Saúde e, em menor proporção, Ciências Humanas e Sociais Aplicadas com o programa Sociedade da Informação. Alguns desses programas, como é o caso desses quatro em que houve um maior enquadramento dos temas das pesquisas, resultaram de ações já desenvolvidas pelo CNPq, com participação da comunidade científica na sua elaboração.

Sendo assim, apesar do alto conteúdo aplicado e tecnológico dos programas, e do fato de serem bolsas concedidas enquanto demanda espontânea, essa alta convergência de temas em algumas áreas mostra a relação estreita entre desenvolvimento científico e tecnológico ou a efetivação de um "modelo misto de desenvolvimento científico e tecnológico" (Sobral e Trigueiro, 1994).

No entanto, embora o desenvolvimento social esteja nos objetivos gerais da política científica e tecnológica atual, as Ciências Humanas, nas

4 Os resultados referentes às áreas de Engenharia e de Lingüística e Letras ainda não estão concluídos. 
quais se inclui a Sociologia, e os temas sociais, estão tendo pouco espaço neste quadro. Pode-se observar na tabela em anexo que as pesquisas das Ciências Agrárias têm um alto grau de convergência com os temas do programa de Agronegócio (em torno de $90 \%$ em todas as modalidades de bolsas) e, em proporções insignificantes, com outros programas.

As pesquisas das Ciências Biológicas convergem em torno de $50 \%$ a $80 \%$ com temas do programa de Biotecnologia e Recursos Genéticos e se enquadram aproximadamente, de $20 \%$ a $45 \%$, com os temas do programa de Fomento à Pesquisa em Saúde.

As pesquisas das Ciências da Saúde convergem com os temas do programa de Fomento à Pesquisa em Saúde em aproximadamente 85\% a 90\%, com os temas do programa de Biotecnologia e Recursos Genéticos em 7\% a 18\%, e com os temas do programa Sociedade da Informação de 1\% a 29\%.

Já o programa com o qual as pesquisas das Ciências Humanas, das Ciências Sociais Aplicadas, e das Ciências Exatas e da Terra mais convergem é o programa Sociedade da Informação. Porém, as proporções não são tão altas como nas outras áreas já citadas: de 15\% a 30\%, nas Ciências Humanas; de 23\% a 30\%, nas Ciências Sociais Aplicadas e de 9\% a 26\% nas Ciências Exatas e da Terra. As pesquisas das Ciências Exatas e da Terra e das Ciências Humanas também convergem com os temas do programa de Fomento à Pesquisa em Saúde, porém, em menor proporção do que nas outras grandes áreas: de 9\% a 13\% e de 9\% a 16\%.

\section{O desafio das Ciências Sociais}

Qual tem sido, então, o desafio das Ciências Sociais e da Sociologia diante desse contexto? Serem relevantes, embora não necessariamente prioritárias. Ou seja, produzirem conhecimento reflexivo, mesmo que os seus temas não estejam incluídos entre as prioridades da política científica e tecnológica. Pois a Sociologia tem uma tradição de reflexão sobre as ins- 
tituições e sobre as transformações sociais em curso.

As origens da Sociologia se deram num contexto social em ebulição. Surgia uma nova sociedade industrial, moderna, capitalista, na qual os clássicos da Sociologia analisaram o aparecimento de novas classes sociais, a divisão social do trabalho e novas formas de solidariedade, a emergência da autonomia individual associada à consolidação de solidariedade social, a constituição do Estado Nacional, a substituição da religião pela ciência, etc. Também, já nessa época, falava-se da contribuição do desenvolvimento científico e tecnológico para a acumulação de capital, para a racionalização e secularização da sociedade e, no caso da Sociologia mais especificamente foi destacada a sua importância para a reforma moral da sociedade.

A questão que gostaria de abordar hoje aqui é que a sociedade contemporânea também está em transformação e que as Ciências Sociais têm contribuído muito com a reflexão tanto sobre questões clássicas, porém redimensionadas, como sobre questões emergentes, apontando, inclusive alguns caminhos para as políticas.

São as Ciências Sociais, às vezes junto com outras disciplinas, que têm principalmente estudado as novas relações de trabalho, os novos processos educacionais e de produção do conhecimento, as formas de violência, a estrutura rural, a emancipação feminina, as novas formas de constituição das identidades individuais e coletivas, os vários tipos de expressão das desigualdades sociais, os impactos sociais da revolução científica e tecnológica, entre outras. Mas esses temas nem sempre constam especificamente do PPA, dos Institutos do Milênio ou dos Fundos Setoriais, embora possam ser abordados tangencialmente.

Porém, é, de novo, às Ciências Sociais que cabe desvendar esses meandros do desenvolvimento científico e tecnológico que revelam, por um lado, a natureza social da ciência e da tecnologia e, por outro, a complexidade do social, pois a ciência e a tecnologia se localizam no campo da ação social, um campo de saberes em disputa, de exercícios de poder e de 
lutas por hegemonia. Ou seja, elas se situam num campo de conflitos, pois há uma diversidade de necessidades sociais a que estão vinculadas (Figueiredo, 1989). Por isso, têm autonomia, mas não distância da sociedade.

Ou seja, nascemos em transformação, vivemos em transformação, estamos em transformação. Somos uma instituição que analisa as transformações e nos transformamos também.

Distribuição percentual das pesquisas apoiadas pelo CNPq segundo enquadramento em programas prioritários, área de conhecimento e modalidade de bolsa

\begin{tabular}{|c|c|c|c|c|c|}
\hline & \multirow{2}{*}{$\mathrm{P}$} & \multicolumn{4}{|c|}{ Modalidade de bolsa } \\
\hline & & 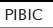 & Mestrado & Doutorado & $P Q$ \\
\hline & B & 5,1 & 2,2 & 3,3 & 1,6 \\
\hline & c & 92,4 & 92,2 & 96,7 & 94,9 \\
\hline & $P$ & 2,3 & - & - & 4,0 \\
\hline & Sociedade da Informação & 7,0 & 2,2 & - & 0,4 \\
\hline \multirow{4}{*}{ Biológicas } & Biotecnologia para Recursos Genéticos & 54,4 & 79,1 & 67,1 & 70,7 \\
\hline & Ciência e Tecnologia para Agronegócios & 16,3 & 24,5 & 20,5 & 19,3 \\
\hline & Fomento à Pesquisa em Saúde & 44,1 & 22,3 & 31,5 & 45,1 \\
\hline & Sociedade da Informação & 3,6 & 1,4 & 2,7 & 2,6 \\
\hline \multirow{4}{*}{ Exatas e da Terra } & Biotecnologia para Recursos Genéticos & 0,5 & 0,5 & - & - \\
\hline & Ciência e Tecnologia para Agronegócios & 1,8 & 2,0 & $\cdot$ & 1,4 \\
\hline & Fomento à Pesquisa em Saúde & 12,9 & 10,3 & 8,6 & 2,4 \\
\hline & Sociedade da Informação & 15,8 & 25,5 & 9,4 & 13,1 \\
\hline \multirow{4}{*}{ Humanas } & Biotecnologia para Recursos Genéticos & 0,5 & 0,6 & 1,2 & 1,1 \\
\hline & Ciência e Tecnologia para Agronegócios & 1,8 & 1,7 & - & 2,9 \\
\hline & Fomento à Pesquisa em Saúde & 8,9 & 16,1 & 4,9 & 9,4 \\
\hline & Sociedade da Informação & 28,9 & 16,7 & 14,6 & 34,3 \\
\hline \multirow{4}{*}{ Saúde } & Biotecnologia para Recursos Genéticos & 17,7 & 7,4 & 15,5 & 15,1 \\
\hline & Ciência e Tecnologia para Agronegócios & 8,2 & 8,5 & 1,7 & 0,5 \\
\hline & Fomento à Pesquisa em Saúde & 92,8 & 78,7 & 86,2 & 85,9 \\
\hline & Sociedade da Informação & 11,0 & 13,8 & 29,3 & 1,1 \\
\hline \multirow{4}{*}{ Sociais Aplicadas } & Biotecnologia para Recursos Genéticos & - & - & - & - \\
\hline & Ciência e Tecnologia para Agronegócios & 1,6 & 4,3 & - & 1,7 \\
\hline & Fomento à Pesquisa em Saúde & 0,4 & 3,2 & - & 6,6 \\
\hline & Sociedade da Informação & 28,5 & 27,7 & 30,4 & 23,3 \\
\hline
\end{tabular}


Sociologias, Porto Alegre, ano 6, no 11, jan/jun 2004, p. 220-237

\section{Referências}

ETZKOWITZ, H.; LEYDESDORFF, L. Emergence of a Triple - Helix of University Industry-government relations. In: Science and Public Policy, 23 (5), 1996.

FERNANDES, A. M.; SOBRAL, F.A. da F. (Orgs). Colapso da Ciência e da Tecnologia no Brasil. Rio de Janeiro: Relume-Dumará, 1994.

FIGUEIREDO, V. Produção Social da Tecnologia. São Paulo: EPU, 1989.

GIBBONS, M. et al. The New Production of knowledge: the dynamics of science in contemporary societies. London: Sage, 1994.

KNORR-CETINA, K. Scientific comunities or transepistemics arenas of research? A critique of quasi economic models of science. In: Social Studies of Science, n. 12, 1982.

LATOUR, B.; WOLGAR, S. A Vida de Laboratório. Rio de Janeiro: Relume-Dumará, 1997.

LATOUR, B. Le Métier de chercheur: regard d'un anthropologue. Paris: INRA, 1995.

MACIEL, M. L.; SOBRAL, F. A. da F.; TRIGUEIRO, M. (Orgs). A Alavanca de Arquimedes. Brasília: Paralelo 15, 1997.

MERTON, R. K. Sociologia: Ideologia e Estrutura. São Paulo: Mestre Jou, 1968.

NEVES, C. B. Ciência e Tecnologia no Brasil. In: SOARES, M. S. A. Educação Superior no Brasil. Brasília: CAPES, 2002.

SOBRAL, F. A. da F.; TRIGUEIRO, M. G. S. Limites e potencialidades da base técnico-científica. In: FERNANDES, A M.; SOBRAL, F. (Orgs.). Colapso da Ciência e da Tecnologia no Brasil. Rio de Janeiro: Relume-Dumará, 1994.

SOBRAL, F. A. da F. Para onde vai a pós-graduação brasileira? In: MACIEL, M.; SOBRAL, F. A. da F.; TRIGUEIRO, M. (Orgs). A Alavanca de Arquimedes. Brasília: Paralelo 15, 1997. 
SOBRAL, F. A. da F. A Universidade e o Novo Modo de Produção do Conhecimento. In: Caderno CRH, no 34, jan./jun. 2001.

SOBRAL, F.A. da F. A Economia e a Física no Brasil: campos científicos ou transcientíficos. In: BAUMGARTEN, M. (Org.). A Era do Conhecimento: Matrix ou Ágora? Porto Alegre/Brasília: Ed. Universidade/UFRGS/Ed. UnB, 2001.

Recebido: 05/08/2003

Aceite final: 30/12/2003

\section{Resumo}

O artigo procura analisar o contexto amplo do desenvolvimento científico e tecnológico que, ao mesmo tempo, torna possível e limita a produção do conhecimento em Ciências Sociais. Para isso, apresenta algumas idéias de estudos contemporâneos sobre as novas tendências do desenvolvimento científico e tecnológico e aborda as políticas recentes de ciência e tecnologia, sob a ótica dessas idéias, enfatizando a transição para um modelo misto do desenvolvimento científico e tecnológico. Em seguida, mostra resultados de pesquisa que indicam essas novas tendências e, finalmente, discute os desafios das Ciências Sociais diante desse quadro.

Palavras-chave: Ciências Sociais, modelo misto de desenvolvimento científico e tecnológico, prioridade. 